\title{
Contents, Vol. 85, 1935
}

\section{Inhaltsverzeichnis.}

\section{Eigenarbeiten.}

Bresgen, C, Zur Kenntnis der Knochenbildung in der Aderhaut 217 Fischer, Fr., Über die Ursachen bitemporaler Hemianopsie

bei Schwangerschaft 88

Fischer, I., Zur Vorgeschichte der Wiener Augenkliniken . 326

Fiszman, T., Das Pfeifen mit den Tränenwegen 27

Hossmann, E., Über einen Fall von Erkrankung der Anhangsgebilde der Augen auf dem Boden einer Agranulocytose 117 Huber, Oth., Stammbaum mit seltener Häufung von Katarakt 310 Imre, J'., Weitere Angaben zur Elektrolyse gegen Netzhautablösung $\quad 336$

Kaleff, R., Über Bindehaut- und Mundschleimhautplastik bei der Elliotschen Operation 73

Kramer, R., Bemerkungen zur sogenannten Velonoskiaskopie von Trantas 300

Krassó, J., Diagnostik und Behandlung tuberkulöser Erkrankungen des Auges mittels subkonjunktivaler Tebeprotininjektionen (hierzu Tafel I) 271 Kyrieleis, W., Zur Technik und Wirkungsweise der Eigenbluteinspritzung in die Vorderkammer bei tuberkulöser Iridozyklitis nach Schieck 16 Löjjler, A., Ergebnisse der Hambur gschen Behandlung bei tabischer Sehnervenatrophie 196 Löwenstein, A., Die perkutane Tuberkulinbehandlung bei Augenkrankheiten mit Hilfe von Glas- oder Schmirgelpapierabreibung 191

Mehlmack, F., Ein Beitrag zur Frage der Embolie der Arteria centralis retinae und ihrer Äste 213

Ohm, J., Der optokinetische Nystagmus bei hysterischer Blindheit 65

Peters, A., Zur ,,Dehnsucht des Auges” 1 Pillat, A., Zur Frage der postoperativen Netzhautablösung nach Zyklodialyse 5

Schläpjer, H., Über das Wesen des Blendungsschmerzes . 221

Schmidt, R., Gitterfasern in der Bindehaut 24

Schlodtmann, W., Zwei seltene Krankheitsbilder bei einem

Patienten (Vollständige Abreißung der Iris bei nichtperforierender stumpier Verletzung und Keratocele bei Dystrophia marginalis corneae) 
Tertsch, R., Über das Verhalten der Leukozytenzahl unter

Einfluß des japanischen Tuberkuloseantigens AO als

Methode zur Bestimmung der Immunitätsverhältnisse 314

- IV -

Seit $\beta$

Velhagen, K., Die Bedeutung des blinden Fleckes bei der Be-

urteilung der Stauungspapille 288

Vogt, A., Zur Geschichte der Katholyse der Netzhautablösung 2 Wilczek, M., Ein Fall der

Netzhautspaltung (Retinoschisis)

mit einer Öffnung 108

Aus der Praxis für die Praxis.

Brockmeyer, G., Über das Dunkelzittern kleiner Kinder.

Von Dr. G. Brockmeyer-Bottrop 121

Goldfeder, A., Über einen durch Bluttransfusion geheilten Fallvon schweren Sehstörungen nach

Blutverlust. VonPriv.-Doz. Dr. A. Goldfeder in Charkow 31

Berichte über die ophthalmologische Literatur.

Sehbahn, Auge und Nervenkrankheiten. Von Prof. Dr. W. Kyrieleis

in Hamburg 36

Entwicklungsgeschichte des Auges, Mißbildungen, Anthropologie.

Von R. Seefelder-Innsbruck 132

Normale und pathologische Anatomie. Von Priv.-Doz. Dr. Rudolf

Schneider- Graz 153

Physiologische und psychologische Optik. Von Priv.-Doz. Dr. H.

Gaste $3 / 8$ er-Innsbruck 223

Vererbung und Auge. Von Prof. Dr. Franceschetti-Gení .... 243 Entstehung und Klinik der

Refraktionsanomalien, Sehschärfe, Ak-

kommodation. Von Doz. Dr. K. §afáf in Wien 338

Gesellschaftsberichte.

Ophthalmologische Gesellschaft in Wien. Sitzung am 15. Okt. 1934163

Sitzung am 19. Nov. 1934261 Sitzung am 10. Dez. 1934347

Vereinigung mitteldeutscher Augenärzte. 38. Tagung am 16. und

17. Juni $1934 \frac{1}{8}$ Jeda 57

25. wissenschaftliche Sitzung der augenärztlichen Vereinigung desOberbergamtsbezirks

Dortmund am 22. September $1934 \mathrm{~m}$ derAugenheilanstalt Mülheim/Ruhr 170

Jahresversammlung der Ungarischen Ophthalmologischen Gesellschaft. Sitzung am 1. bis 3. Juni

$1934 \times 77$

Verhandlungen der Schwedischen Gesellschaft der Augenärzte.

21. Jahressitzung in Stockholm am 3. Juni $1934 \quad 2(\mathrm{H}$

Bayerische Augenärztliche Vereinigung. Neunte Tagung in Regens-

burg am 11. November $193435^{\circ}$

Diagnose und Therapie 61, 188, 268, 362

Wilhelm Reis $\uparrow$. Von Prof. Dr. P. Junius in Bonn/Rh 366

Personalien 64, 190, 270, 367

Buchbesprechung 63,368

S. Sacbxegister zu Band $8 \delta \quad 369$

Namenreg $1 / 8$ ter zu Band $85 \quad 374$ 
